BRIEF COMMUNICATION

\title{
Bilateral Symmetry in Finer Lines of Increased Density
}

\author{
STANLEY M. GARN AND RAYMOND S. BABY \\ Center for Human Growth and Development, University of Michigan, \\ Ann Arbor, Michigan 48104 and The Ohio Historical Museum, \\ Columbus, Ohio
}

\begin{abstract}
Radiogrammetric comparisons of the left and right femora of adult archaic Indian Knoll specimens from Kentucky show bilateral correspondence in more than $50 \%$ of "key" lines of increased radiographic density, indicating the symmetrical nature of the original developmental events.
\end{abstract}

Broad bands and heavy lines of increased radiographic density make their bilateral appearance following phosphorous poisoning, ingestion of heavy metals, hypervitaminosis $D$, steroid therapy of dwarfism and transfusion therapy of anemias (Garn et al., '68). Bilateral appearances of bone strata and spicules within the marrow cavity have also been observed in tubular bones from prehistoric populations (McHenry, '68). Such clinical observations and explorations in paleo-anthropology raise the possibility that much of the finer structure of bones may constitute an individual historical record (cf. Garn and Schwager, '67). But to what extent do the fine markings occupy corresponding positions and exhibit truly symmetrical appearances on left and right limbs respectively?

In attempting to resolve these questions, we started with the simple superimposition of paired left and right limb radiographs, using a high-intensity dense-film viewer. This superimposition method proved satisfactory for the larger transverse lines, (cf. Garn et al., '68, cover illustration ), but was unsuited to finer detail. Accordingly, we resorted to the use of transparent acetate or polyester overlays, (Kodak Transparent Sleeves) in order to outline "key" or marker lines, on each of the limb views separately, and then superimposing the left-limb overlay on the right-limb radiograph, and vice versa.

Using the overlay technique on the left and right lateral femoral views of 34 adults from the Indian Knoll skeletal collection, we attempted to identify corresponding nonepiphyseal lines of increased radio- graphic density. Of the 34 archaic American Indian skeletons, 28 exhibited multiple lines of increased density on the distal third of the femora, corresponding in radiographic appearance to the spicules and strands of bone previously pictured (Garn et al., '68, figs. $31,34,35$ and cover). Of the 28 pairs of femora so identifed by key or reference lines, 18 individuals exhibited one or more matching lines on both sides. Thus the majority of skeletalized individuals so studied, had corresponding finer lines on both sides. In all, a total of 140 key nonepiphyseal lines was included in the bilateral comparison, and 74 (37 pairs) were spatially corresponding. So better than $50 \%$ of the lines in the distal third of the Indian Knoll femora also exhibited correspondences.

Correspondence between lines on the left and right sides is shown for one subject (24-235) in figure 1. Here a "mirroring" technique is employed, matching the corresponding anterior halves of the left and right limb lateral projections. The arrangement of the lines within the marrow cavity is clear, as is the near-correspondence of the epiphyseal lines as well. In similar fashion, but using a slightly different approach, bilateral correspondence of lines in a second specimen (24-311) is shown in the second photographic illustration (fig. 2). Here the anterior half of one femur and the photographically-reversed posterior half of the other femur are mounted so as to simulate a single complete lateral femoral view. The lines of increased density in figure 2 resemble the spicules demonstrated in tibial and femo- 


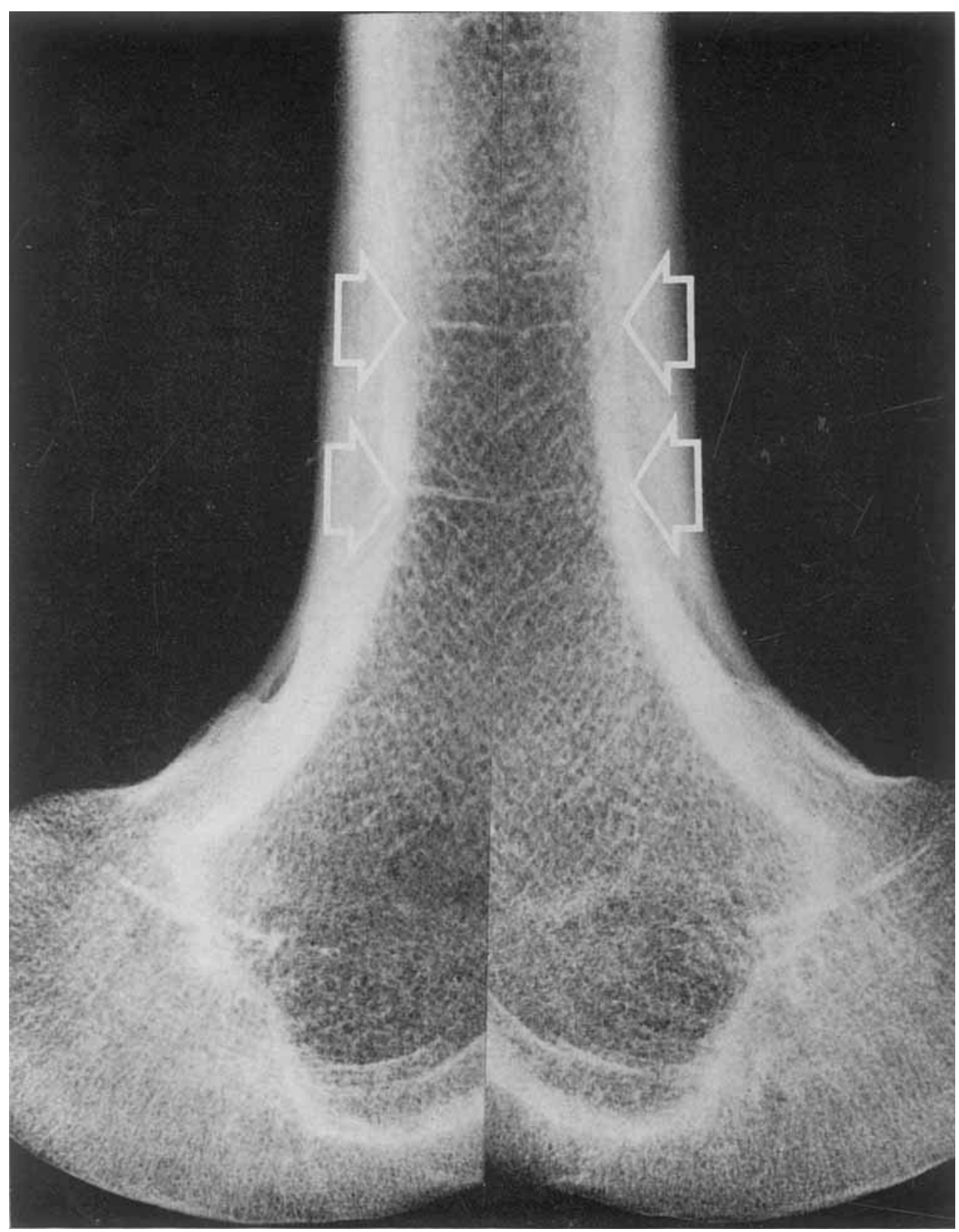

Fig. 1 Bilateral correspondence of fine nonepiphyseal lines in the distal aspect of the femur as shown by the "mirroring" technique. Here the lateral views of the left femur (right) and right femur (left) of a young adult Indian Knoll male show closely matching fine transverse lines of increased density within the marrow cavity. Specimen 24-235.

ral sections, and correspond closely in position (see illustration).

Corresponding lines on the left and right femora may also be used to measure rel- ative bone growth at the distal ends. If the location of all corresponding lines, including epiphyseal lines, is plotted as in figure 3 , a longitudinal growth record 


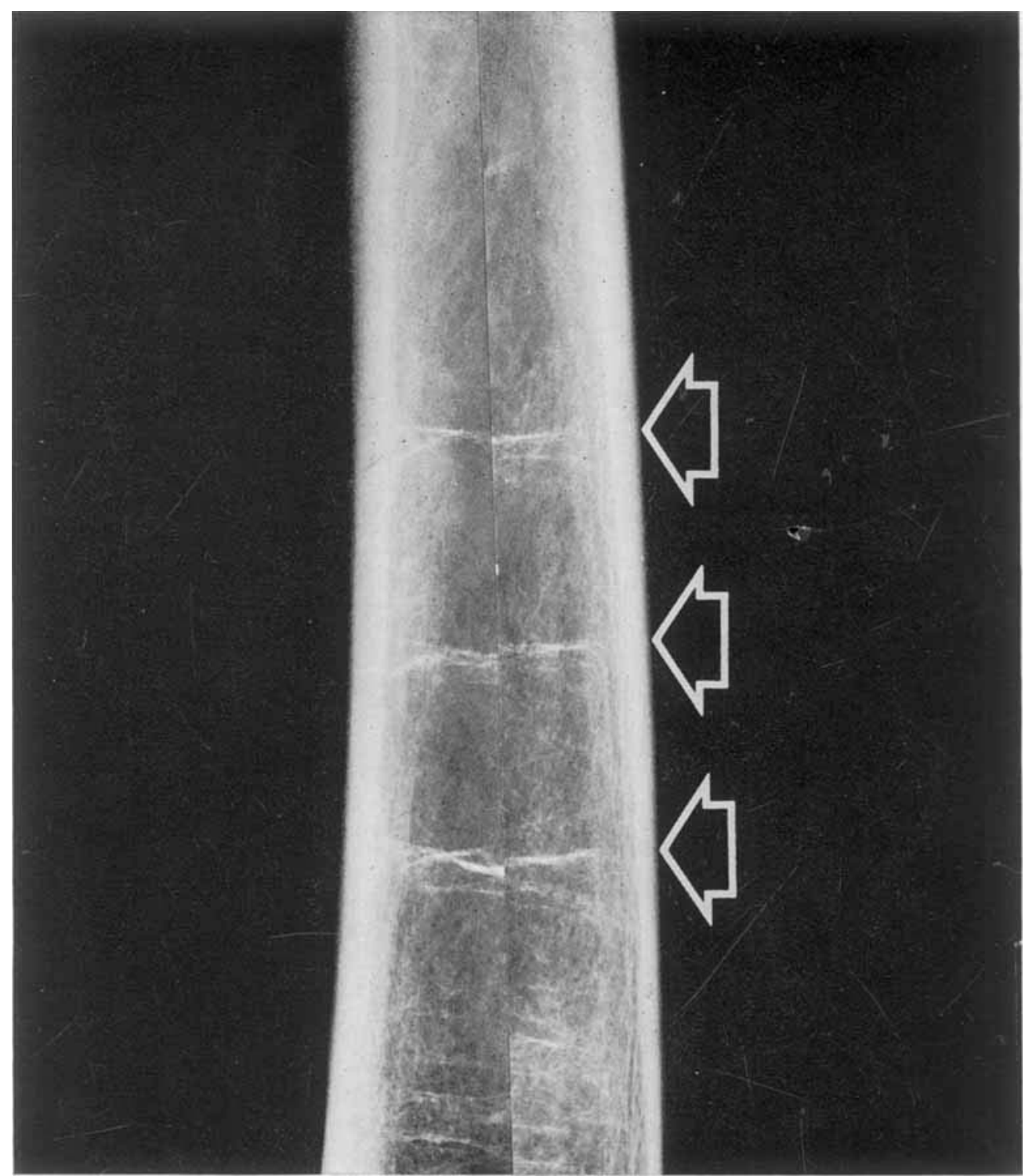

Fig. 2 Bilateral correspondence of multiple lines of increased density as shown by the imagereversal technique, picturing the anterior half of the left femur (left) and the reversed posterior half of the right femur (right). In this composite print of Indian Knoll female 24-211, the lines resemble those in Garn et al., '68, figs. 30, 31, 33 and 34. The distance between the lines closely approximates one year's growth at the distal femur.

emerges as pictured. Though the individual in figure 3 is long-dead, and the operational approach is therefore crosssectional, the corresponding lines reflect the slight inequality in limb length, and partial recovery at the end of childhood, providing a serial, longitudinal growth record retrospectively.

Critical comparison of left and right femoral radiographs suggests that the majority of lines of increased radiographic density reflect systemic rather than purely 


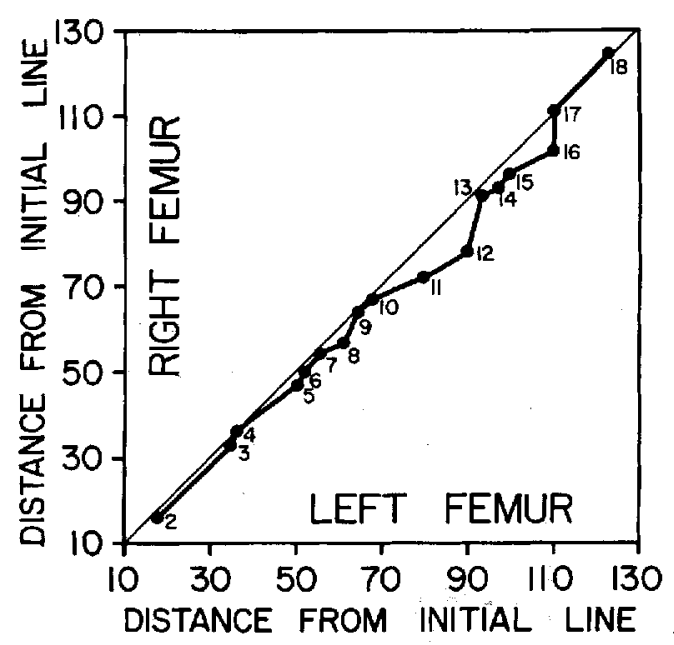

Fig. 3 Distances (in $\mathrm{mm}$ ) of 18 corresponding lines of increased density in the distal third of the left femur (abscissa) and the right femur (ordinate) of a young archaic adult female from the Indian Knoll collection. The relative positions of the lines indicate a transient inequality of femoral growth during childhood, with the left femur slightly longer throughout.

local influences. Such correspondence may well be less in a generally-older population sample, following resorption. Comparable correspondence might not be expected in in vivo studies, using screen-type films, screens and grids that lose finer detail. Furthermore it is clear that relatively narrow lines on one side, well under $1 \mathrm{~mm}$, may correspond to lines over $1 \mathrm{~mm}$ in thickness on the other side. However, it is evident that such lines persist for many years (cf. Garn and Schwager, '67), they are primarily within the marrow cavity (Garn et al., '68; McHenry, '68), they are bilateral in their placement, they constitute growth records of the individuals under study (Garn, Schwager and Hempy, '68) and they even provide a historical record of unequal limb growth.

\section{ACKNOWLEDGMENTS}

This study was supported by grant AM-13378 from the National Institutes of Health, using radiographs taken by Yvonne French and measurements and matching accomplished by Judy Schweitzer. The manuscript was completed by Shirley M. Garrett.

\section{LITERATURE CITED}

Garn, S. M., H. O. Hempy, III, and P. M. Schwager 1968 Measurement of localized bone growth employing natural markers. Am. J. Phys. Anthrop., 28: 105-108.

Garn, S. M., and P. M. Schwager 1967 Age dynamics of persistent transverse lines in the tibia. Am. J. Phys. Anthrop., 27: 375-377.

Garn, S. M., F. N. Silverman, K. P. Hertzog and C. G. Rohmann 1968 Lines and bands of increased density. Med. Radiog. and Photog., 44: 58-89.

McHenry, H. 1968 Transverse lines of arrested growth in the bones of prehistoric populations. Am. J. Phys. Anthrop., 29: 1-17. 